\title{
Bifurcation of Limit Cycles and Center Conditions for Two Families of Kukles-Like Systems with Nilpotent Singularities
}

\author{
Peiluan Li, ${ }^{1,2}$ Yusen $\mathrm{Wu}^{2}$ and Xiaoquan Ding ${ }^{2}$ \\ ${ }^{1}$ School of Mathematics and Computer, Hunan Normal University, Changsha, Hunan 410083, China \\ ${ }^{2}$ School of Mathematics and Statistics, Henan University of Science and Technology, Luoyang, Henan 471023, China \\ Correspondence should be addressed to Yusen Wu; wuyusen621@126.com
}

Received 26 May 2013; Revised 26 August 2013; Accepted 28 August 2013

Academic Editor: Mark McKibben

Copyright (c) 2013 Peiluan Li et al. This is an open access article distributed under the Creative Commons Attribution License, which permits unrestricted use, distribution, and reproduction in any medium, provided the original work is properly cited.

We solve theoretically the center problem and the cyclicity of the Hopf bifurcation for two families of Kukles-like systems with their origins being nilpotent and monodromic isolated singular points.

\section{Introduction}

One of the main open problems in the qualitative theory of planar analytic differential systems is the case when the singular point is monodromic (the orbits move around the singular point). A monodromic point of an analytic system is either a center (i.e., a singular point with a punctures neighborhood filled with periodic orbits) or a focus (i.e., a singular point with a neighborhood where all the orbits are spirals which arrive at the equilibrium point in forward or backward time). The problem of distinguishing when a monodromic singular point is either a center or a focus is called the center problem.

The so-called center problem for planar vector fields has been intensively and extensively studied over the last century and is also closely related to Hilbert's 16 th problem. The singular point can be elementary or not in the sense whether the corresponding Jacobian matrix has zero eigenvalues. If the eigenvalues of the quoted matrix are imaginary with real part null, the singular point may be a focus or a center, which is known as the celebrated Poincaré-Lyapunov center problem and has been theoretically solved by Poincaré [1] and Liapunov [2]. If the matrix of the linear part at the singular point has its two eigenvalues equal to zero, but it is not identically null, by Andreev [3] we know what is the behavior of the solutions in a neighborhood of the singular point, except if it is a center of a focus (nilpotent center problem).

Yet for the bifurcation of limit cycles and center problem of nilpotent singular points in a planar vector field, its intrinsic dynamics is still far away from understanding due to the complexity and technical difficulties. Therefore, it is natural to restrict our study to nilpotent singularities. An analytic system of differential equations in the plane having an isolated nilpotent singularity, in some suitable coordinates, can be written as

$$
\begin{gathered}
\dot{x}=y+X(x, y), \\
\dot{y}=Y(x, y),
\end{gathered}
$$

with $X(x, y)$ and $Y(x, y)$ real analytic functions without constant nor linear terms defined in a certain neighborhood of the singularity.

The study of nilpotent singularities and their unfolding for vector fields is important not only for mathematical interest but also for practical reasons. To solve the finite cyclicity problem in the second part of Hilbert's 16th problem, Dumortier et al. $[4,5]$ presented an impressive list of 121 graphics that occur in quadratic systems, among which many are nilpotent cases, studied in [6-11]. On the other hand, the center problem for nilpotent singularities is another interesting issue and has been partially solved; see [3,12-19], and so forth.

Liu and Li [20] originated a method in studying the center problem of third-order nilpotent singularities by integral factor method, where a new definition of the focal values, quasi-Lyapunov constants, is provided. At the same time, the equivalence of quasi-Lyapunov constants and focal values is proved. A linear recursive formula for computing quasiLyapunov constants is also derived. Afterward, they proved 
that if the third-order nilpotent origin is a $m$-order weakened focus, then, by a small perturbation for the unperturbed system, there exist $m$ limit cycles in a neighborhood of the origin. Meanwhile, the origin becomes an elementary critical point and two complex singular points.

In the present paper, we are interested in the study of two families of Kukles-like systems with third-order nilpotent part of the form $\left(y,-2 x^{3}\right)$ and, respectively, nonlinear part given by quartic and quintic homogeneous polynomial with an isolated singular point at the origin; that is,

$$
\begin{gathered}
\frac{d x}{d t}=y \\
\frac{d y}{d t}=-2 x^{3}+b_{40} x^{4}+b_{31} x^{3} y \\
+b_{22} x^{2} y^{2}+b_{13} x y^{3}+b_{04} y^{4} \\
\frac{d x}{d t}=y \\
\frac{d y}{d t}=-2 x^{3}+b_{50} x^{5}+b_{41} x^{4} y \\
+b_{32} x^{3} y^{2}+b_{23} x^{2} y^{3}+b_{14} x y^{4}+b_{05} y^{5}
\end{gathered}
$$

This paper will be organized as follows. In Section 2 we summarize some general definitions and results about thirdorder nilpotent singularities. In Section 3 we present the conditions for third-order nilpotent singularities to be a center and give the proof of the cyclicity for system (2). In the last section, we present the parallel results for system (3).

\section{The Formula to Compute the Quasi-Lyapunov Constants}

The origin is a third-order monodromic critical point if and only if the system is of the following form:

$$
\begin{aligned}
\frac{d x}{d t} & =y+\mu x^{2}+\sum_{i+2 j=3}^{\infty} a_{i j} x^{i} y^{j} \\
& =X(x, y), \\
\frac{d y}{d t} & =-2 x^{3}+2 \mu x y+\sum_{i+2 j=4}^{\infty} b_{i j} x^{i} y^{j} \\
& =Y(x, y) .
\end{aligned}
$$

The quasi-Lyapunov constant is defined and a way of computing them is provided in [20].

Theorem 1. For any positive integer s and a given number sequence

$$
\left\{c_{0 \beta}\right\}, \quad \beta \geq 3,
$$

one can construct successively the terms with the coefficients $c_{\alpha \beta}$ satisfying $\alpha \neq 0$ of the formal series

$$
\begin{aligned}
M(x, y) & =y^{2}+\sum_{\alpha+\beta=3}^{\infty} c_{\alpha \beta} x^{\alpha} y^{\beta} \\
& =\sum_{k=2}^{\infty} M_{k}(x, y)
\end{aligned}
$$

such that

$$
\begin{gathered}
\left(\frac{\partial X}{\partial x}+\frac{\partial Y}{\partial y}\right) M-(s+1)\left(\frac{\partial M}{\partial x} X+\frac{\partial M}{\partial y} Y\right) \\
=\sum_{m=3}^{\infty} \omega_{m}(s, \mu) x^{m},
\end{gathered}
$$

where for all $k, M_{k}(x, y)$ is a $k$-homogeneous polynomial of $x, y$ and $s \mu=0$.

It is easy to see that (7) is linear with respect to the function $M$, so that we can easily find in [20] the following recursive formulae for the calculation of $c_{\alpha \beta}$ and $\omega_{m}(s, \mu)$.

Theorem 2. For $\alpha \geq 1, \alpha+\beta \geq 3$ in (6) and (7), $c_{\alpha \beta}$ can be uniquely determined by the recursive formula

$$
c_{\alpha \beta}=\frac{1}{(s+1) \alpha}\left(A_{\alpha-1, \beta+1}+B_{\alpha-1, \beta+1}\right) .
$$

For $m \geq 1, \omega_{m}(s, \mu)$ can be uniquely determined by the recursive formula

$$
\omega_{m}(s, \mu)=A_{m, 0}+B_{m, 0},
$$

where

$$
\begin{gathered}
A_{\alpha \beta}=\sum_{k+j=2}^{\alpha+\beta-1}[k-(s+1)(\alpha-k+1)] \\
\times a_{k j} c_{\alpha-k+1, \beta-j}, \\
B_{\alpha \beta}=\sum_{k+j=2}^{\alpha+\beta-1}[j-(s+1)(\beta-j+1)] \\
\times b_{k j} c_{\alpha-k, \beta-j+1} .
\end{gathered}
$$

Notice that in (10), one sets

$$
\begin{gathered}
c_{00}=c_{10}=c_{01}=0, \\
c_{20}=c_{11}=0, \quad c_{02}=1, \\
c_{\alpha \beta}=0, \quad \text { if } \alpha<0 \text { or } \beta<0 .
\end{gathered}
$$

We see from Theorem 1 that if the origin of system (4) is $s$-class or $\infty$-class, then, by choosing $\left\{c_{\alpha \beta}\right\}$, such that

$$
\omega_{2 k+1}(s, \mu)=0, \quad k=1,2, \ldots,
$$

we can obtain a solution group of $\left\{c_{\alpha \beta}\right\}$ of (12); thus, we have

$$
\lambda_{m}=\frac{\omega_{2 m+4}(s, \mu)}{2 m-4 s-1} .
$$

Clearly, the recursive formulas presented by Theorem 2 are linear with respect to all $c_{\alpha \beta}$. Therefore, it is convenient to realize the computations of quasi-Lyapunov constants by using computer algebraic system like MATHEMATICA. 


\section{Center Conditions and Limit Cycles of System (2)}

By Theorems 1 and 2, we directly have the following.

Lemma 3. Assume that s is a natural number. One can derive a power series (6) for system (2) under which (7) is satisfied, where

$$
\begin{aligned}
& c[0,0]=0, \quad c[1,0]=0, \quad c[0,1]=0 \text {, } \\
& c[2,0]=0, \quad c[1,1]=0, \quad c[0,2]=1 \text {. }
\end{aligned}
$$

In addition, for any natural numbers $\alpha, \beta, c[\alpha, \beta]$ is given by the following recursive formula:

$$
\begin{aligned}
c[\alpha, \beta]=( & b_{40}(1+s)(2+\beta) c[-5+\alpha, 2+\beta] \\
& +b_{31}(1-(1+s)(1+\beta)) c[-4+\alpha, 1+\beta] \\
& +2(1+s)(2+\beta) c[-4+\alpha, 2+\beta] \\
& +b_{22}(2-(1+s) \beta) c[-3+\alpha, \beta] \\
& +b_{13}(3-(1+s)(-1+\beta)) \\
& \times c[-2+\alpha,-1+\beta] \\
& +b_{04}(4-(1+s)(-2+\beta)) \\
& \times c[-1+\alpha,-2+\beta]) \times(\alpha(s+1))^{-1}
\end{aligned}
$$

and, for any natural number $m, \omega_{m}$ is given by the following recursive formula:

$$
\begin{aligned}
\omega_{m}= & -b_{40}(1+s) c[-4+m, 1] \\
& +b_{31} c[-3+m, 0]+2(1+s) c[-3+m, 1] \\
& +b_{22}(3+s) c[-2+m,-1] \\
& +b_{13}(3+2(1+s)) c[-1+m,-2] \\
& +b_{04}(4+3(1+s)) c[m,-3] .
\end{aligned}
$$

The straightforward computing according to Lemma 3 with MATHEMATICA gives

$$
\begin{gathered}
\omega_{6}=0 \\
\omega_{7}=\frac{1}{2}\left(b_{31}-2 b_{31} s+6 c_{03}+6 s c_{03}\right) \\
\omega_{8} \sim-\frac{1}{10} b_{40} b_{31}(-3+4 s) \\
\omega_{9} \sim-2 b_{13}(-1+s) .
\end{gathered}
$$

To make $\omega_{9}=0$, we will consider two different cases: $b_{13}=0$ and $s=1$.
Case $1\left(b_{13}=0\right)$. Taking expression (13) into account, the first four quasi-Lyapunov constants of system (2) are given by

$$
\begin{gathered}
\lambda_{1}=0, \\
\lambda_{2} \sim \frac{1}{10} b_{40} b_{31}, \\
\lambda_{3} \sim \frac{1}{21} b_{31} b_{22}, \\
\lambda_{4} \sim-\frac{2}{9} b_{31} b_{04} .
\end{gathered}
$$

Now we can prove the following result.

Theorem 4. In this case, the origin of system (2) is a center if and only if the first four quasi-Lyapunov constants vanish; that is, one of the following two series is satisfied:

$$
\begin{gathered}
b_{31}=b_{13}=0, \\
b_{40}=b_{22}=b_{13}=b_{04}=0 .
\end{gathered}
$$

Proof. It is direct to obtain conditions (19) and (20) by imposing $\lambda_{i}=0, i=1,2,3,4$.

On the other hand, when condition (19) holds, system (2) goes over to

$$
\begin{gathered}
\frac{d x}{d t}=y, \\
\frac{d y}{d t}=-2 x^{3}+b_{40} x^{4}+b_{22} x^{2} y^{2}+b_{04} y^{4},
\end{gathered}
$$

whose vector field is symmetric with respect to the $x$-axis. When condition (20) holds, system (2) goes over to

$$
\begin{gathered}
\frac{d x}{d t}=y, \\
\frac{d y}{d t}=x^{3}\left(-2+b_{31} y\right),
\end{gathered}
$$

whose vector field is symmetric with respect to the $y$-axis. This ends the proof.

Case $2(s=1)$. Taking expression (13) into account, the first five quasi-Lyapunov constants of system (2) are given by

$$
\begin{gathered}
\lambda_{1}=0, \\
\lambda_{2} \sim \frac{1}{10} b_{40} b_{31}, \\
\lambda_{3} \sim \frac{1}{105}\left(5 b_{31} b_{22}+9 b_{40} b_{13}\right), \\
\lambda_{4} \sim \frac{1}{6300}\left(680 b_{22} b_{13}-1400 b_{31} b_{04}-117 b_{40}^{3} b_{13}\right), \\
\lambda_{5} \sim \frac{1}{8085000} b_{13} \\
\times\left(476000 b_{04}-286495 b_{40}^{2} b_{22}+18018 b_{40}^{5}\right) .
\end{gathered}
$$

For the same reason as before, we obtain the following. 
Theorem 5. In this case, the origin of system (2) is a center if and only if the first five quasi-Lyapunov constants vanish; that is, the following series is satisfied:

$$
b_{40}=b_{22}=b_{04}=0 .
$$

Proof. It is direct to obtain conditions (24) by imposing $\lambda_{i}=$ $0, i=1,2,3,4,5$.

On the other hand, when condition (24) holds, system (2) goes over to

$$
\begin{gathered}
\frac{d x}{d t}=y \\
\frac{d y}{d t}=x\left(-2 x^{2}+b_{31} x^{2} y+b_{13} y^{3}\right),
\end{gathered}
$$

whose vector field is symmetric with respect to the $y$-axis. Then, we have proved the theorem.

It is not hard to see that condition (20) is a particular case of condition (24); in summary, the following result characterizes the center of system (2).

Theorem 6. System (2) has a center at the origin if and only if one of the following two sets of conditions holds:

(I) $b_{31}=b_{13}=0$,

(II) $b_{40}=b_{22}=b_{04}=0$.

Remark 7. Indeed, in the light of the theory of [21], the origin is not only a center but also an analytic center under condition (I).

Referring to the expressions given in (18) and (23), we have the following.

Lemma 8. The origin of system (2) is a fine focus of maximum order five. Further, it is of order five if and only if

$$
b_{40}=b_{31}=b_{22}=0, \quad b_{13} b_{04} \neq 0 .
$$

Consider the perturbed system of (2)

$$
\begin{gathered}
\frac{d x}{d t}=\delta(\varepsilon) x+y \\
\frac{d y}{d t}=2 \delta(\varepsilon) y-2 x^{3}+b_{21}(\varepsilon) x^{2} y+b_{40}(\varepsilon) x^{4} \\
+b_{31}(\varepsilon) x^{3} y+b_{22}(\varepsilon) x^{2} y^{2}+b_{13} x y^{3}+b_{04} y^{4}
\end{gathered}
$$

Theorem 9. Under a small perturbation of system (2), for a small parameter $\delta$, in a neighborhood of the origin of system (27), there exist exactly 5 small amplitude limit cycles enclosing the elementary node $\mathrm{O}(0,0)$.

The next example shows this fact.

Example 10. Take

$$
\begin{aligned}
& \delta(\varepsilon)=-\varepsilon^{30}, \\
& b_{21}(\varepsilon)=\varepsilon^{20}, \\
& b_{40}(\varepsilon)=\varepsilon^{6},
\end{aligned}
$$

$$
\begin{aligned}
& b_{31}(\varepsilon)=-\varepsilon^{6}, \\
& b_{22}(\varepsilon)=-\varepsilon^{2}, \\
& b_{13}=b_{04}=1 .
\end{aligned}
$$

Straightforward computations by using expression (23) give the first 5 quasi-Lyapunov constants of the origin of system (27):

$$
\begin{gathered}
\lambda_{1}=\frac{1}{3} \varepsilon^{20}+o\left(\varepsilon^{20}\right) \approx 0.333333 \varepsilon^{20}+o\left(\varepsilon^{20}\right), \\
\lambda_{2} \sim-\frac{1}{10} \varepsilon^{12}+o\left(\varepsilon^{12}\right)=-0.1 \varepsilon^{12}+o\left(\varepsilon^{12}\right), \\
\lambda_{3} \sim \frac{3}{35} \varepsilon^{6}+o\left(\varepsilon^{6}\right) \approx 0.0857143 \varepsilon^{6}+o\left(\varepsilon^{6}\right), \\
\lambda_{4} \sim-\frac{34}{315} \varepsilon^{2}+o\left(\varepsilon^{2}\right) \approx-0.107937 \varepsilon^{2}+o\left(\varepsilon^{2}\right), \\
\lambda_{5} \sim \frac{68}{1155}+o(1) \approx 0.0588745+o(1) .
\end{gathered}
$$

Thereby, for $0<\varepsilon \ll 1$, system (27) has 5 limit cycles $\Gamma_{k}$ : $r=\tilde{r}\left(\theta, h_{k}(\varepsilon)\right)$ in a small neighborhood of the origin, where $h_{k}(\varepsilon)=o\left(\varepsilon^{k}\right), k=1,2,3,4,5$.

\section{Center Conditions and Limit Cycles of System (3)}

Similar to Lemma 3, we have the following.

Lemma 11. Assume that s is a natural number. One can derive a power series (6) for system (3) under which (7) is satisfied, where

$$
\begin{aligned}
& c[0,0]=0, \quad c[1,0]=0, \quad c[0,1]=0, \\
& c[2,0]=0, \quad c[1,1]=0, \quad c[0,2]=1 .
\end{aligned}
$$

In addition, for any natural numbers $\alpha, \beta, c[\alpha, \beta]$ is given by the following recursive formula:

$$
\begin{aligned}
c[\alpha, \beta]=( & b_{50}(1+s)(2+\beta) c[-6+\alpha, 2+\beta] \\
& +b_{41}(1-(1+s)(1+\beta)) c[-5+\alpha, 1+\beta] \\
& +b_{32}(2-(1+s) \beta) c[-4+\alpha, \beta] \\
& +2(1+s)(2+\beta) c[-4+\alpha, 2+\beta] \\
& +b_{23}(3-(1+s)(-1+\beta)) \\
& \times c[-3+\alpha,-1+\beta] \\
& +b_{14}(4-(1+s)(-2+\beta)) \\
& \times c[-2+\alpha,-2+\beta] \\
& +b_{05}(5-(1+s)(-3+\beta)) \\
& \times c[-1+\alpha,-3+\beta]) \times(\alpha(s+1))^{-1},
\end{aligned}
$$


and, for any natural number $m, \omega_{m}$ is given by the following recursive formula:

$$
\begin{aligned}
\omega_{m}= & -b_{50}(1+s) c[-5+m, 1] \\
& +b_{41} c[-4+m, 0]+b_{32}(3+s) c[-3+m,-1] \\
& +2(1+s) c[-3+m, 1] \\
& +b_{23}(3+2(1+s)) c[-2+m,-2] \\
& +b_{14}(4+3(1+s)) c[-1+m,-3] \\
& +b_{05}(5+4(1+s)) c[m,-4] .
\end{aligned}
$$

Taking expression (13) into account, the first four quasiLyapunov constants of system (3) are given by

$$
\begin{aligned}
\lambda_{1} & =0, \\
\lambda_{2} & \sim \frac{1}{5} b_{41}, \\
\lambda_{3} & \sim \frac{2}{7} b_{23}, \\
\lambda_{4} & \sim \frac{4}{3} b_{05} .
\end{aligned}
$$

Hence, we arrive at the following.

Theorem 12. System (3) has a center at the origin if and only if the following set of conditions holds:

$$
\text { (III) } b_{41}=b_{23}=b_{05}=0 \text {. }
$$

Proof. It is direct to obtain condition (III) by imposing $\lambda_{i}=0$, $i=1,2,3,4$.

On the other hand, when condition (III) is satisfied, system (3) reduces to

$$
\begin{gathered}
\frac{d x}{d t}=y, \\
\frac{d y}{d t}=x\left(-2 x^{2}+b_{50} x^{4}+b_{32} x^{2} y^{2}+b_{14} y^{4}\right),
\end{gathered}
$$

whose vector field is symmetric with respect to the origin. The claim follows.

Remark 13. Indeed, in the light of the theory of [21], the origin is not only a center but also an analytic center under condition (III).

We obtain the following by expression (33).

Lemma 14. The origin of system (3) is a fine focus of maximum order four. Further, it is of order four if and only if

$$
b_{41}=b_{23}=0, \quad b_{05} \neq 0 \text {. }
$$

Consider the perturbed system of (3):

$$
\begin{gathered}
\frac{d x}{d t}=\delta(\varepsilon) x+y, \\
\frac{d y}{d t}=2 \delta(\varepsilon) y-2 x^{3}+b_{21}(\varepsilon) x^{2} y+b_{50} x^{5} \\
+b_{41}(\varepsilon) x^{4} y+b_{32} x^{3} y^{2}+b_{23}(\varepsilon) x^{2} y^{3} \\
+b_{14} x y^{4}+b_{05} y^{5} .
\end{gathered}
$$

Theorem 15. Under a small perturbation of system (3), for a small parameter $\delta$, in a neighborhood of the origin of system (36), there exist exactly 4 small amplitude limit cycles enclosing the elementary node $\mathrm{O}(0,0)$.

Example 16. Take

$$
\begin{gathered}
\delta(\varepsilon)=\varepsilon^{20}, \\
b_{21}(\varepsilon)=-\varepsilon^{12}, \\
b_{41}(\varepsilon)=\varepsilon^{6}, \\
b_{23}(\varepsilon)=-\varepsilon^{2}, \\
b_{05}=1 .
\end{gathered}
$$

Straightforward computations by using expression (33) give the first 4 quasi-Lyapunov constants of the origin of system (36):

$$
\begin{gathered}
\lambda_{1}=-\frac{1}{3} \varepsilon^{12}+o\left(\varepsilon^{12}\right) \approx-0.333333 \varepsilon^{12}+o\left(\varepsilon^{12}\right), \\
\lambda_{2} \sim \frac{1}{5} \varepsilon^{6}+o\left(\varepsilon^{6}\right)=0.2 \varepsilon^{6}+o\left(\varepsilon^{6}\right), \\
\lambda_{3} \sim-\frac{2}{7} \varepsilon^{2}+o\left(\varepsilon^{2}\right) \approx-0.285714 \varepsilon^{2}+o\left(\varepsilon^{2}\right), \\
\lambda_{4} \sim \frac{4}{3}+o(1) \approx 1.33333+o(1) .
\end{gathered}
$$

Thereby, for $0<\varepsilon \ll 1$, system (36) has 4 limit cycles $\Gamma_{k}$ : $r=\widetilde{r}\left(\theta, h_{k}(\varepsilon)\right)$ in a small neighborhood of the origin, where $h_{k}(\varepsilon)=o\left(\varepsilon^{k}\right), k=1,2,3,4$.

\section{Acknowledgments}

This work is supported in part by the National Nature Science Foundation of China (11101126 and 11271110) and Scientific Research Start-up Fund for Doctoral Scholars of HAUST (09001524).

\section{References}

[1] H. Poincaré, "Mémoire sur les courbes définies par les équations différentielles," Journal de Mathématiques, vol. 37, pp. 375-422, 1881.

[2] M. A. Liapunov, Problème Général De La Stabilité Du Mouvement, vol. 17 of Annals of Mathematics Studies, 1947. 
[3] A. F. Andreev, "Investigation of the behaviour of the integral curves of a system of two differential equations in the neighbourhood of a singular point," American Mathematical Society Translations, vol. 8, pp. 183-207, 1958.

[4] F. Dumortier, R. Roussarie, and C. Rousseau, "Hilbert's 16th problem for quadratic vector fields," Journal of Differential Equations, vol. 110, no. 1, pp. 86-133, 1994.

[5] F. Dumortier, R. Roussarie, and C. Rousseau, "Elementary graphics of cyclicity 1 and 2," Nonlinearity, vol. 7, no. 3, pp. 10011043, 1994.

[6] F. Dumortier, R. Roussarie, and J. Sotomayor, "Bifurcations of cuspidal loops," Nonlinearity, vol. 10, no. 6, pp. 1369-1408, 1997.

[7] Yu. S. Il'yashenko, "Finiteness theorems for limit cycles," Russian Mathematical Surveys, vol. 40, pp. 143-200, 1990.

[8] M. El Morsalani and A. Mourtada, "Degenerate and non-trivial hyperbolic 2-polycycles: appearance of two independant ÉcalleRoussarie compensators and Khovanskiř's theory," Nonlinearity, vol. 7, no. 6, pp. 1593-1604, 1994.

[9] R. Roussarie, "On the number of limit cycles which appear by perturbation of separatrix loop of planar vector fields," Boletim da Sociedade Brasileira de Matemática, vol. 17, no. 2, pp. 67-101, 1986.

[10] R. Roussarie, "Cyclicité finie des lacets et des points cuspidaux," Nonlinearity, vol. 2, no. 1, pp. 73-117, 1989.

[11] H. Zhu and C. Rousseau, "Finite cyclicity of graphics with a nilpotent singularity of saddle or elliptic type," Journal of Differential Equations, vol. 178, no. 2, pp. 325-436, 2002.

[12] M. J. Álvarez and A. Gasull, "Monodromy and stability for nilpotent critical points," International Journal of Bifurcation and Chaos in Applied Sciences and Engineering, vol. 15, no. 4, pp. 1253-1265, 2005.

[13] M. J. Álvarez and A. Gasull, "Generating limit cycles from a nilpotent critical point via normal forms," Journal of Mathematical Analysis and Applications, vol. 318, no. 1, pp. 271-287, 2006.

[14] A. A. Andronov, E. A. Leontovich, I. I. Gordon, and A. G. Mă̌er, Qualitative Theory of Second-Order Dynamic Systems, Wiley, New York, NY, USA, 1973.

[15] J. Chavarriga, H. Giacomin, J. Giné, and J. Llibre, "Local analytic integrability for nilpotent centers," Ergodic Theory and Dynamical Systems, vol. 23, no. 2, pp. 417-428, 2003.

[16] W. W. Farr, C. Li, I. S. Labouriau, and W. F. Langford, "Degenerate Hopf bifurcation formulas and Hilbert's 16th problem," SIAM Journal on Mathematical Analysis, vol. 20, no. 1, pp. 1330, 1989.

[17] V. Mañosa, "On the center problem for degenerate singular points of planar vector fields," International Journal of Bifurcation and Chaos in Applied Sciences and Engineering, vol. 12, no. 4, pp. 687-707, 2002.

[18] R. Moussu, "Symétrie et forme normale des centres et foyers dégénérés," Ergodic Theory and Dynamical Systems, vol. 2, no. 2, pp. 241-251, 1982.

[19] F. Takens, "Singularities of vector fields," Institut des Hautes Études Scientifiques, no. 43, pp. 47-100, 1974.

[20] Y. Liu and J. Li, "On third-order nilpotent critical points: integral factor method," International Journal of Bifurcation and Chaos in Applied Sciences and Engineering, vol. 21, no. 5, pp. 1293-1309, 2011.

[21] T. Liu, L. Wu, and F. Li, "Analytic center of nilpotent critical points," International Journal of Bifurcation and Chaos in Applied Sciences and Engineering, vol. 22, Article ID 1250198, 8 pages, 2012. 


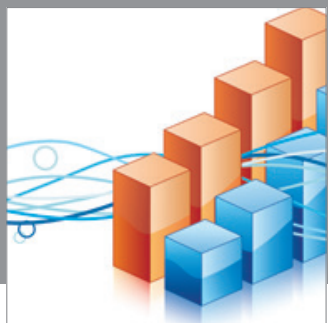

Advances in

Operations Research

mansans

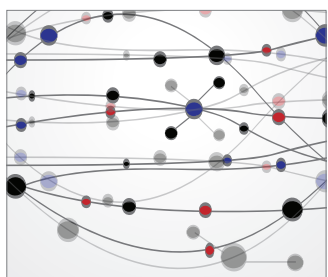

The Scientific World Journal
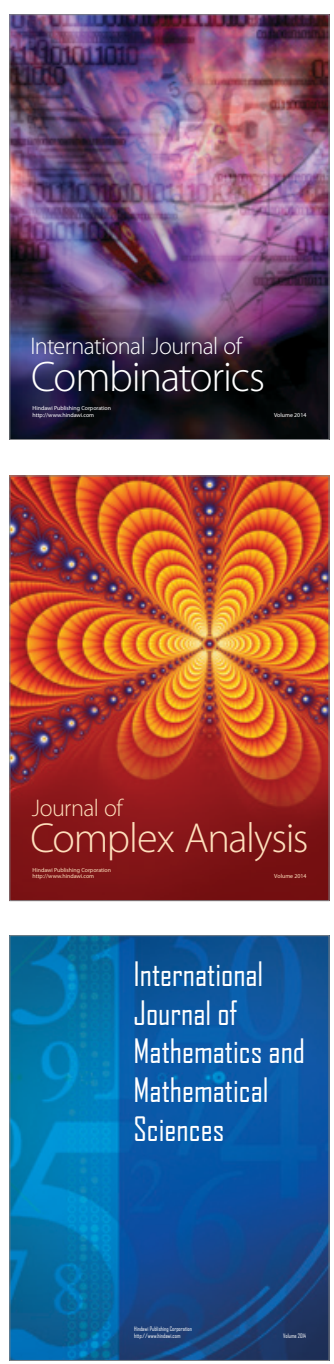
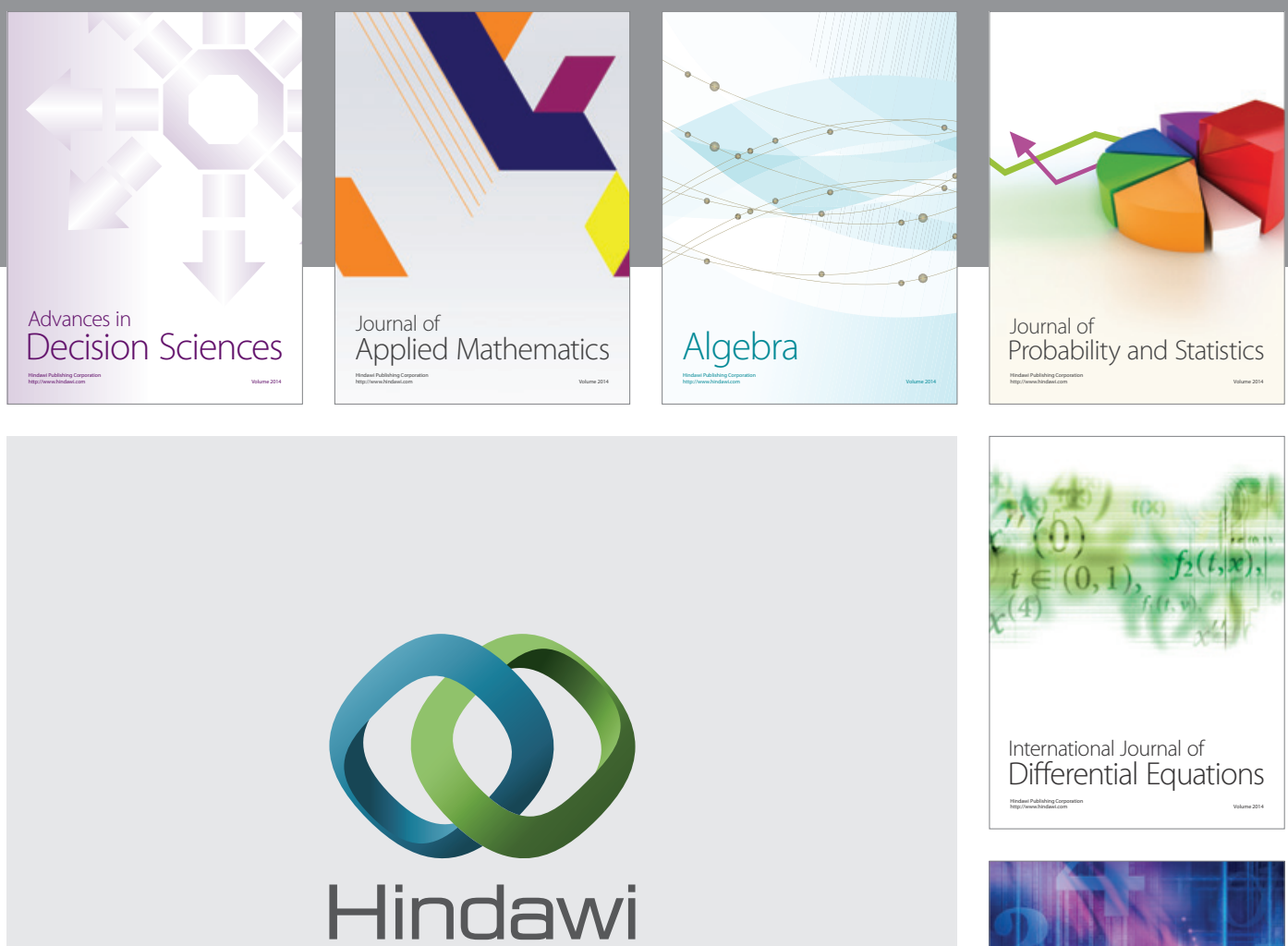

Submit your manuscripts at http://www.hindawi.com
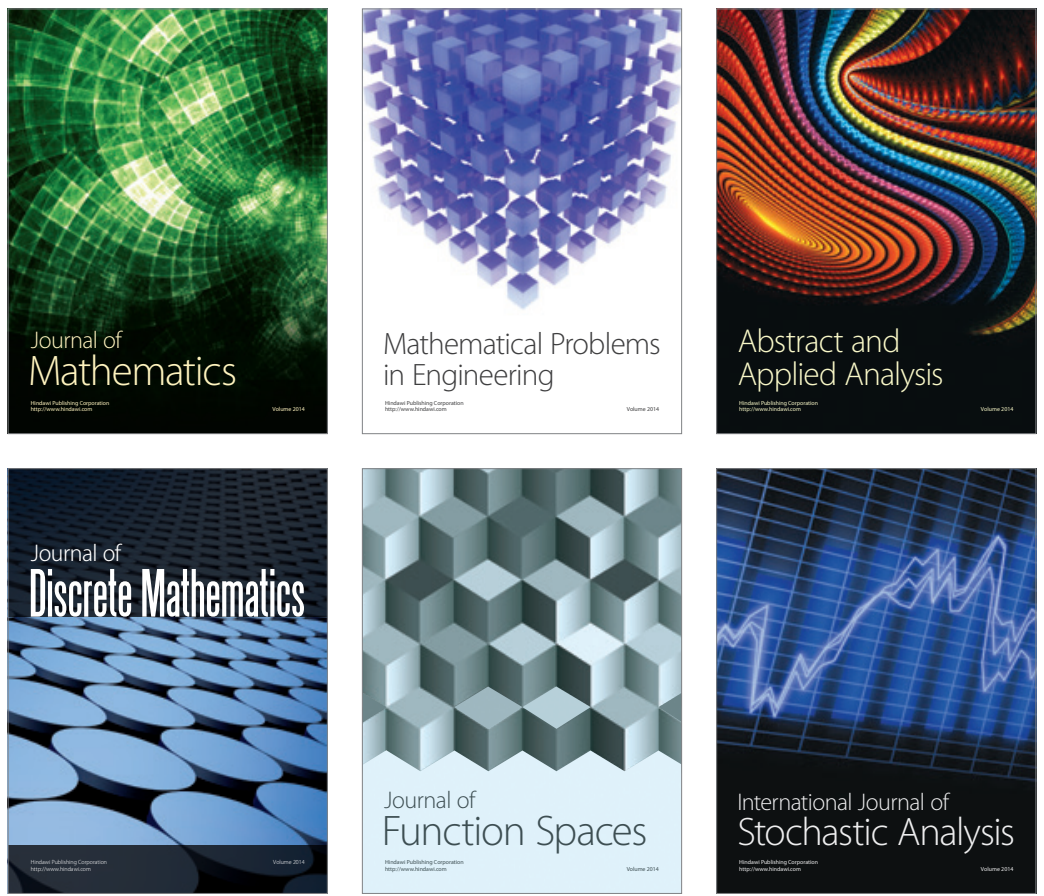

Journal of

Function Spaces

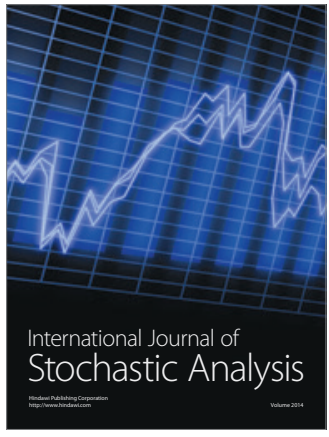

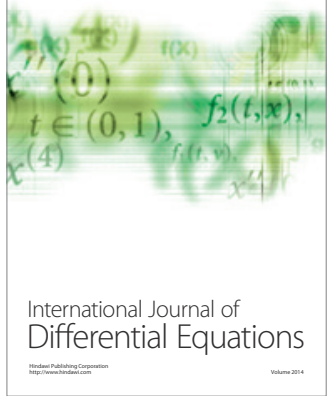
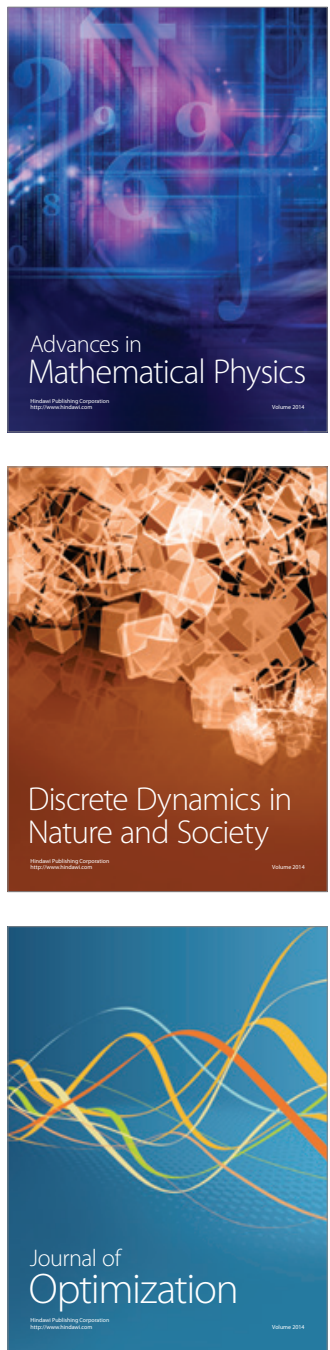